\title{
Hard and Soft Adjusting of a Parameter With Its Known Boundaries by the Value Based on the Experts' Estimations Limited to the Parameter
}

\author{
Vadim V. Romanuke (Professor, Khmelnitskiy National University)
}

\begin{abstract}
Adjustment of an unknown parameter of the multistage expert procedure is considered. The lower and upper boundaries of the parameter are counted to be known. A key condition showing that experts' estimations are satisfactory in the current procedure is an inequality, in which the value based on the estimations is not greater than the parameter. The algorithms of hard and soft adjusting are developed. If the inequality is true and its both terms are too close for a long sequence of expert procedures, the adjusting can be early stopped. The algorithms are reversible, implying inversion to the reverse inequality and sliding up off the lower boundary.
\end{abstract}

Keywords - Expert procedure; Experts' estimations; Law of large numbers; Parameter adjustment; Tolerance.

\section{Problems of AdJusting UnKNown PARAMETERS IN EXPERT PROCEDURES}

Expert procedures are needful part in estimating and evaluating a great deal of attributes or parameters of natural, behavioral, social, economic, and technical processes [1], [2]. Those attributes and parameters cannot be deduced directly, because fundamental laws which might have described them and their structural logic are unknown or unreliable. While estimating, an expert procedure may have its own parameters regulating the experts' judgments [3], [4]. These ones are not deduced all the more. Thus, unknown parameters are adjusted. The adjustment is, factually, some kind of manual optimization, where the optimum is a close-to-best parameter's value. This optimum provides the stronger framework of an expert procedure [3], [5], [6]. If without adjustment, the results of an expert procedure and its consensus value (object) come poor, unfounded, and frail [1], [2], [5]. A crucial problem of the adjustment is that there is no strict theory which could lead to at least the parameter's confidence interval [7]. Practically, any new case in a field of study implies a slightly different technique of adjustment being itself another problem. So, a sub-theory should be developed for every group of the similar study field cases.

\section{APPROACHES to PARAMETER AdJUSTMENT}

Commonly, the parameters of expert procedures are set by experience. They are naively changed if an appropriate convention is established [8], [9]. Such conventions concern weighing experts' estimations, setting the total (minimal or maximal) number of experts, confining the periods of decision making (expert's judgment), etc. While being adjusted, the parameter's value is changed according to those methods bearing a resemblance to linear or simple dichotomy [3], [5],
[8], [10], [11]. These are considerably rough decisions not regarding different importances of the neighboring values of the being adjusted parameter. This, nonetheless, should not be confused with determining the unknown (uncertain) parameters of mathematical models while they are being identified [3], [12], [13]. Parametric identification is referred to mathematical modeling dealing with observations and statistics [3], [7], [12], [14], [15], whereas parameter adjustment issues from expert knowledge and estimation procedures relying on experience.

\section{GOAL AND TASKS}

The goal is to develop an algorithm of adjusting the unknown parameter of the multistage expert procedure so that the corresponding feature of experts' estimations (evaluations) could be compared to the adjusted parameter. The boundaries of the parameter are counted to be known. Thus, the adjustment is equivalent to removing an interval uncertainty whose severity increases as the interval boundaries are located wider [5], [7], [16], [17]. Eventually, an example of applying the developed algorithm is given.

\section{PRELIMINARY CONVENTIONS}

Let $\lambda$ be a value of the parameter to be adjusted. The lower boundary of the parameter is $\lambda_{\min }$, and its upper boundary is $\lambda_{\text {max }}$; both are known real numbers. Thus,

$$
\lambda \in\left[\lambda_{\min } ; \lambda_{\max }\right]
$$

The parameter is adjusted through a series of expert procedures whose number is $Q_{\max }$ by $Q_{\max } \in \mathbb{N}$; although the case $Q_{\max }=1$ is rare, it cannot be excluded. After the $q$-th expert procedure, where the number of the current expert procedure $q=\overline{1, Q_{\max }}$, the value based on the experts' estimations is calculated. The limit of this value is the parameter. For instance, it can be the maximal (minimal, average, etc.) distance between estimations. Denote the value based on the experts' estimations by $\rho$, which is compared to $\lambda$. Let the inequality

$$
\rho \leqslant \lambda
$$

be the key condition showing that experts' estimations are satisfactory in the current procedure. 


\section{HARD AdJUSTING}

Before taking on the first procedure, the mean of the segment $\left[\lambda_{\min } ; \lambda_{\max }\right]$ is taken to be the parameter value:

$$
\lambda=\frac{\lambda_{\max }+\lambda_{\min }}{2}
$$

If inequality (1) is false, then, for the next procedure, the mean of the segment $\left[\rho ; \lambda_{\max }\right]$ is taken:

$$
\lambda=\frac{\rho+\lambda_{\max }}{2} .
$$

Otherwise, the parameter value is moved to the left, and the new one is taken in the mean of the segment $[\rho ; \lambda]$, i. e.,

$$
\lambda_{\text {new }}=\frac{\rho+\lambda}{2} \text { and } \lambda=\lambda_{\text {new }}
$$

for an intermediate value $\lambda_{\text {new }}$. Admittedly, if the values $\lambda$ and $\rho$ are too close, nothing is moved. The closeness is understood as the relative distance less than some tolerance $\delta$. If

$$
\frac{\lambda-\rho}{\lambda_{\max }-\lambda_{\min }}<\delta
$$

then the move to the left by (4), i.e., to the center between points $\rho$ and $\lambda$, is not fulfilled because the values $\lambda$ and $\rho$ are too close. Theoretically, in accordance with $(5), \delta \in(0 ; 1)$. For real practice, however, they should be put to $\delta=0.1$, $\delta=0.05, \delta=0.01$, or similar.

Such adjusting can be called a hard one due to the rough decision on the right move (3) when the key condition (1) turns false. A way of $\rho$ calculation is not specified here, so this is a generalized algorithm of hard adjusting. The value of $\lambda$ is treated to have been adjusted just after maximal number of expert procedures $Q_{\max }$ is passed (Fig. 1). Such criterion of adjustment is valid owing to the law of large numbers [5], [7], [14], [18].

\section{HaRd AdJusting With Closeness RepeAt Control}

The hard adjusting algorithm in Fig. 1 regards the closeness of points $\rho$ and $\lambda$ via inequality (5) and some predefined $\delta \in(0 ; 1)$. However, inequality (5) used to control how close $\rho$ to $\lambda$ is, may turn true for a long sequence of expert procedures. And repeat of this closeness is presumed to say that $\rho$ is stable enough and the adjusting is expected to be stopped. In other words, if the closeness of point $\rho$ to $\lambda$ has recurred many times in succession, this can likely be a manifestation of the law of large numbers. In this case, it is needless to continue the expert procedures.

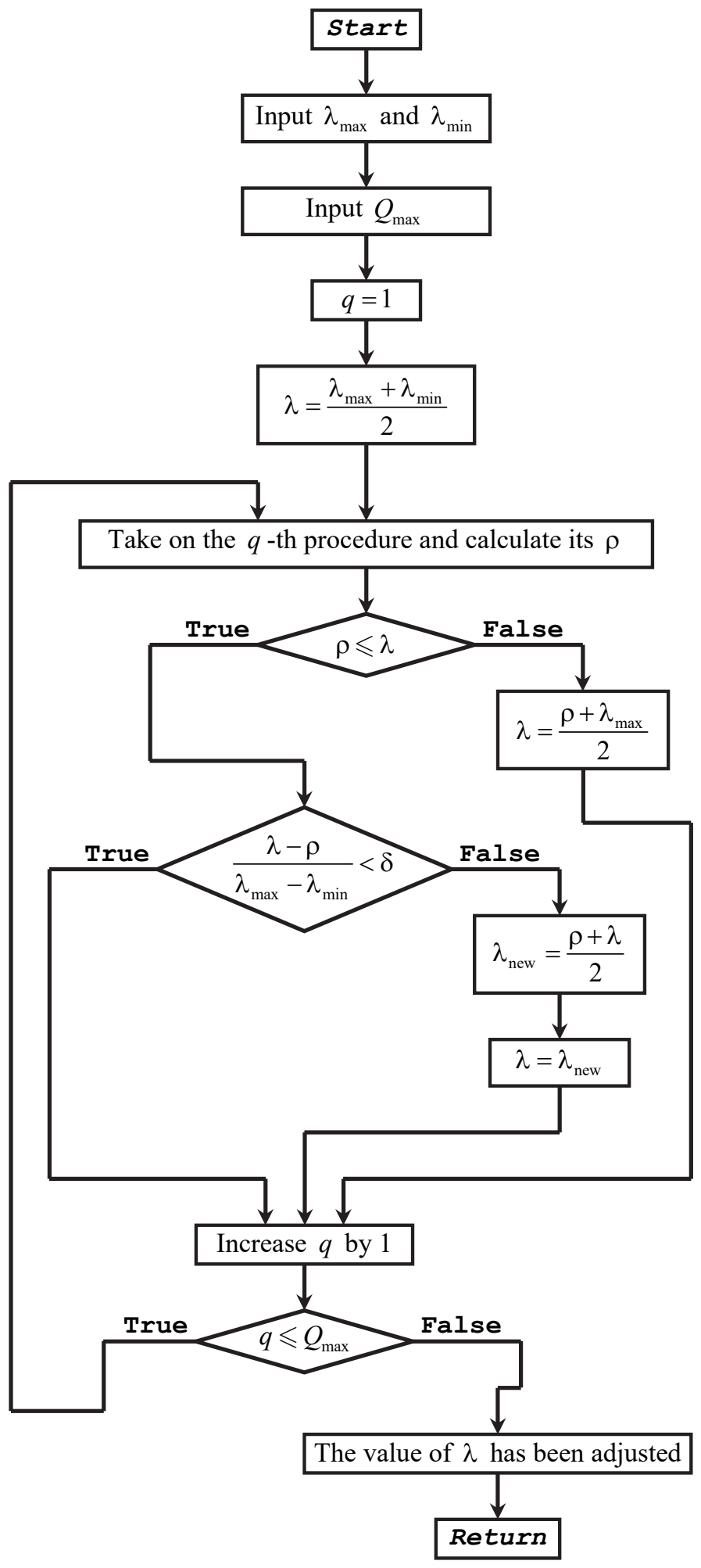

Fig. 1. An algorithm of hard adjusting. At the start, three algorithm parameters are input: real numbers $\lambda_{\min }, \lambda_{\max }$, and positive integer $Q_{\max }$. Tolerance $\delta$ allowing the closeness of $\rho$ to $\lambda$ to be controlled is set beforehand. Note that inequality (5), which is used to control the closeness, may turn true for a long sequence of expert procedures what is presumed to say that $\rho$ is stable enough and the adjusting is expected to be stopped. Neither the way of $\rho$ calculation, nor tolerance $\delta$ is specified here. Once the maximal number of expert procedures $Q_{\max }$ is passed, the value of $\lambda$ is treated to have been adjusted owing to the law of large numbers. 
Let an integer $Q^{*}$ be the ultimate repeat count of the cases when inequality (5) is true. Obviously, $Q^{*} \neq 1$, or else the repeat count will not be started. Besides, $Q^{*}<Q_{\max }$, or else the repeat count is senseless. Another hard adjusting algorithm (Fig. 2) additionally controls the recurring closeness of points $\rho$ and $\lambda$. If the recurrence has been spotted for less than $Q^{*}$ times successively, then, in the next procedure, the repeat counter is set to zero. If it has been spotted for $Q^{*}$ times successively, the value of $\lambda$ is treated to be stable enough and the adjusting is stopped.

\section{SOFT AdJUSTING}

While adjusting is hard, the assignment (3) is ever made if the key condition (1) turns false. This seems to be a drawback when the point $\rho$ is closer to the point $\lambda_{\min }$ rather than to the upper boundary $\lambda_{\max }$. Instead of taking the mean of the segment $\left[\rho ; \lambda_{\max }\right]$, it would be far more rational to take the mean of a subsegment

$$
\left[\rho ; \lambda_{\mathrm{obs}}^{\langle i\rangle}\right] \subset\left[\rho ; \lambda_{\max }\right]
$$

whose right end $\lambda_{\text {obs }}^{\langle i\rangle}$ is the $i$-th obsolete value of $\lambda$, wherein $\lambda_{\text {obs }}^{\langle i+1\rangle}<\lambda_{\text {obs }}^{\langle i\rangle}$ for any positive integer $i$. The obsolescence implies that after having moved towards the left end, a new value of $\lambda$ is taken, and the preceding one is obsolete. The first obsolete value of $\lambda$ is $\lambda_{\text {obs }}^{\langle 1\rangle}=\lambda_{\text {max }}$.

While the parameter's value is adjusted softly, the case when inequality (1) turns false generates smarter conditions to get a new value of $\lambda$. If inequality (1) is false, then, for the next procedure, the inequality

$$
\rho \leqslant \lambda_{\text {obs }}^{\langle i\rangle}
$$

is checked. Obviously, that there always exists such $i \in \mathbb{N}$ that inequality (6) turns true because, anyway,

$$
\rho \leqslant \lambda_{\mathrm{obs}}^{\langle 1\rangle}=\lambda_{\text {max }} .
$$

While (6) is false, the integer $i$ is decreased. Once inequality (6) is true,

$$
\lambda=\frac{\rho+\lambda_{\mathrm{obs}}^{\langle i\rangle}}{2},
$$

if only $\rho$ is not too close to $\lambda_{\text {obs }}^{\langle i\rangle}$ by the current $i$. If

$$
\frac{\lambda_{\mathrm{obs}}^{\langle i\rangle}-\rho}{\lambda_{\max }-\lambda_{\min }}<\delta
$$

then $\rho$ and $\lambda_{\text {obs }}^{\langle i\rangle}$ are too close, and
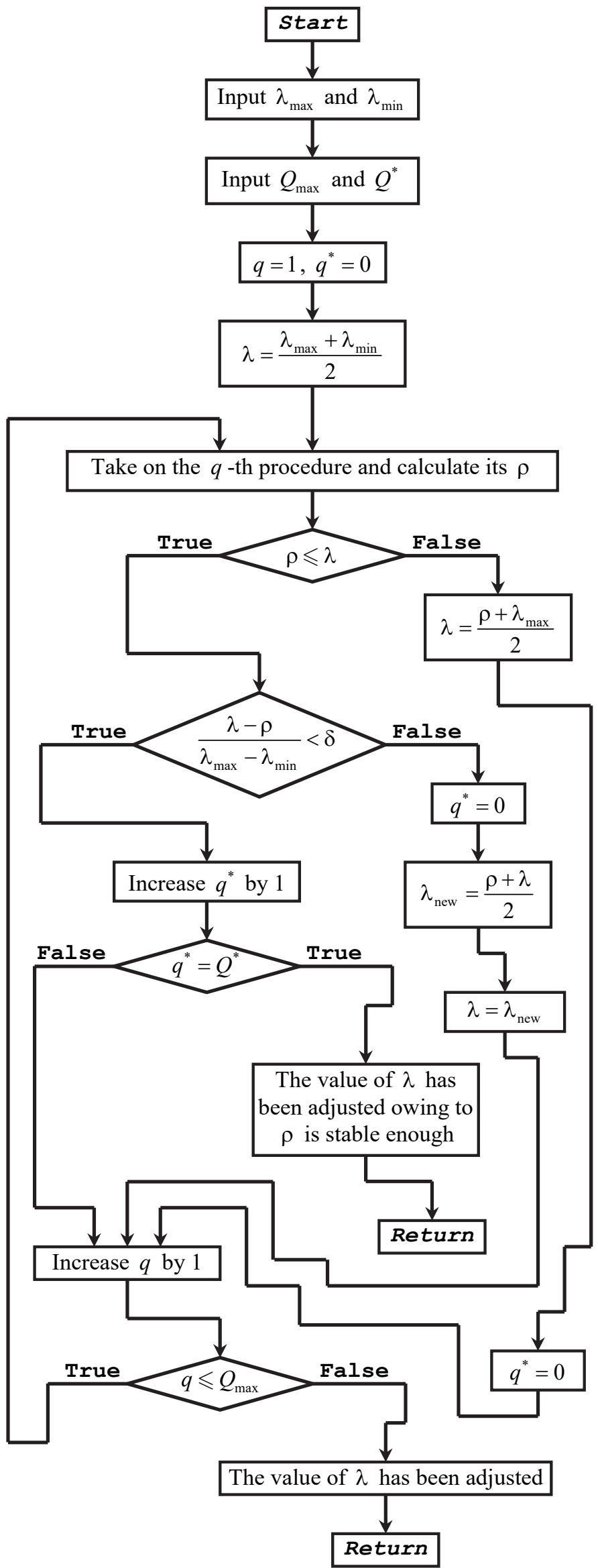

Fig. 2. Another hard adjusting algorithm providing closeness repeat control. There is an adjusting early stop criterion added to the algorithm in Fig. 1. 

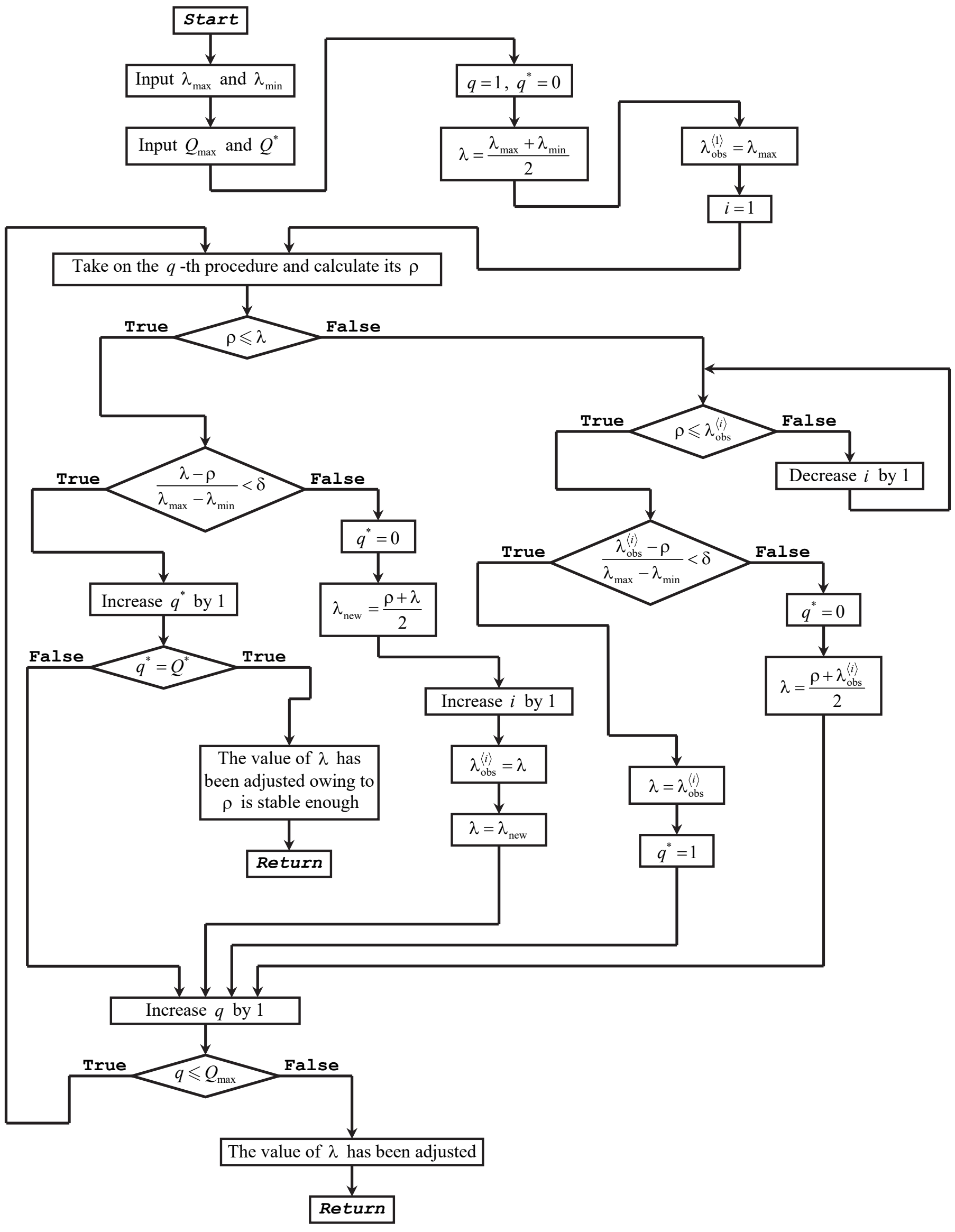

Fig. 3. A soft adjusting algorithm. False inequality (1) gives smarter conditions to get a new $\lambda$. The control of the recurring closeness of $\rho$ to $\lambda$ is maintained to fit the hard adjusting algorithm in Fig. 2. The algorithm stage, where the current expert procedure number is increased by 1, is supplied already with four parents. 


$$
\lambda=\lambda_{\mathrm{obs}}^{\langle i\rangle}
$$

The third algorithm in Fig. 3 has been induced to fit the hard adjusting algorithm in Fig. 2 by maintaining the control of the recurring closeness of $\rho$ to $\lambda$. The algorithm stage, where the current expert procedure number is increased by 1 , is supplied already with four parents unlike the three ones in Figs 1 and 2.

\section{APPLICATION}

The adjusting algorithms in Figs 1-3 are universal. Of course, every group of the similar study field cases holds its own way to calculate $\rho$, to define boundaries $\lambda_{\min }$ and $\lambda_{\text {max }}$, integers $Q_{\max }$ and $Q^{*}$, and tolerance $\delta$. When experts' estimations are point values, the application of any of the developed adjusting algorithms is almost trivial. As an example, consider how they are applied to the studies where experts' estimations are matrices. For the sake of definiteness in exemplification, let $S$ experts make their judgments in the form of $2 \times 2$-matrices whose entries are only ones and zeros. If

$$
\mathbf{B}_{s}=\left[b_{k j}^{\langle s\rangle}\right]_{2 \times 2}
$$

is an estimation of the $s$-th expert, then the average estimation (let it be the consensus) is

$$
\tilde{\mathbf{B}}=\left[\tilde{b}_{k j}\right]_{2 \times 2} \text { by } \tilde{b}_{k j}=\frac{1}{S} \sum_{s=1}^{S} b_{k j}^{\langle s\rangle} \text {. }
$$

A couple of matrices (10) and (11) is distinguished by a distance between them. The distance is

$$
\begin{gathered}
\tilde{\rho}_{s}=\left(\left(\tilde{b}_{11}-b_{11}^{\langle s\rangle}\right)^{2}+\left(\tilde{b}_{12}-b_{12}^{\langle s\rangle}\right)^{2}+\right. \\
\left.+\left(\tilde{b}_{21}-b_{21}^{\langle s\rangle}\right)^{2}+\left(\tilde{b}_{22}-b_{22}^{\langle s\rangle}\right)^{2}\right)^{\frac{1}{2}} .
\end{gathered}
$$

The worst case, producing the severest uncertainty [5], [15], [16], here is

$$
\tilde{\mathbf{B}}=\left[\begin{array}{ll}
1 / 2 & 1 / 2 \\
1 / 2 & 1 / 2
\end{array}\right],
$$

which is possible when $S$ is even. Then the distance (12) reaches its maximum uncertainty value at matrix (13), whatever entries of matrix (10) are:

$$
\tilde{\rho}_{0}=\left(\left(\frac{1}{2}\right)^{2}+\left(\frac{1}{2}\right)^{2}+\left(\frac{1}{2}\right)^{2}+\left(\frac{1}{2}\right)^{2}\right)^{\frac{1}{2}}=1
$$

It is clear that the above-mentioned value based on the experts' estimations is

$$
\rho=\frac{1}{S} \sum_{s=1}^{S} \tilde{\rho}_{s}
$$

Therefore, (15) is limited to (14) as

$$
\tilde{\rho}_{0}=\lambda_{\text {max }}=1 \text {. }
$$

To define $\lambda_{\min }$, consider the "best" case when only a single expert's matrix $\mathbf{B}_{s_{*}}$ exists whose single entry $b_{k_{*} j_{*}}^{\left\langle s_{*}\right\rangle}$ differs from the rest $S-1$ entries which are identical. In other words,

$$
\begin{gathered}
b_{k j}^{\left\langle s_{1}\right\rangle}=b_{k j}^{\left\langle s_{2}\right\rangle} \quad \forall s_{1}=\overline{1, S-1} \text { and } \forall s_{2}=\overline{s_{1}+1, S} \\
\text { by } k \neq k_{*} \text { and } j \neq j_{*},
\end{gathered}
$$

whereas

$$
b_{k_{*} j_{*}}^{\left\langle s_{*}\right\rangle} \neq b_{k_{*} j_{*}}^{\langle s\rangle} \quad \forall s \in\{\overline{1, S}\} \backslash\left\{S_{*}\right\} .
$$

Then

$$
\tilde{b}_{k j}=b_{k j}^{\left\langle s_{*}\right\rangle} \text { by } k \neq k_{*} \text { and } j \neq j_{*} \text {, }
$$

and either

$$
\tilde{b}_{k_{*} j_{*}}=\frac{S-1}{S} \text { by } b_{k_{*} j_{*}}^{\left\langle s_{*}\right\rangle}=0
$$

or

$$
\tilde{b}_{k_{*} j_{*}}=\frac{1}{S} \quad \text { by } \quad b_{k_{*} j_{*}}^{\left\langle s_{*}\right\rangle}=1
$$

Nevertheless, the distance between the consensus and the "special" matrix $\mathbf{B}_{s_{*}}$ is the same:

$$
\tilde{\rho}_{s_{*}}=\sqrt{\left(\tilde{b}_{k_{*} j_{*}}-b_{k_{*} j_{*}}^{\left\langle s_{*}\right\rangle}\right)^{2}}=\sqrt{\left(\frac{S-1}{S}-0\right)^{2}}=\sqrt{\left(\frac{1}{S}-1\right)^{2}}=\frac{S-1}{S} .
$$

And the rest $S-1$ distances are also the same:

$$
\tilde{\rho}_{s}=\sqrt{\left(\tilde{b}_{k_{*} j_{*}}-b_{k_{*} j_{*}}^{\langle s\rangle}\right)^{2}}=\sqrt{\left(\frac{S-1}{S}-1\right)^{2}}=\sqrt{\left(\frac{1}{S}-0\right)^{2}}=\frac{1}{S} .
$$

Finally,

$$
\begin{gathered}
\lambda_{\min }=\frac{1}{S}\left(\tilde{\rho}_{s_{*}}+\sum_{s \in\{1, S\} \backslash\left\{s_{*}\right\}} \tilde{\rho}_{s}\right)= \\
=\frac{1}{S}\left(\frac{S-1}{S}+(S-1) \cdot \frac{1}{S}\right)=\frac{2(S-1)}{S^{2}} .
\end{gathered}
$$

Now, after we have defined the boundaries $\lambda_{\text {min }}=2(S-1) S^{-2}$ and $\lambda_{\text {max }}=1$ by the calculation rule (15), the integers $Q_{\max }$ and $Q^{*}$ and the tolerance $\delta$ remain to be defined. Particularly, tolerance can be set to $\delta=0.1$ or $\delta=0.05$, but it should rather be smaller as the number of experts $S$ increases.

\section{DISCUSSION}

The core algorithmic approach of the hard adjusting demonstrated in Fig. 2 gives the algorithm in Fig. 1 by setting 
$Q^{*}=Q_{\max }$. The soft adjusting rejects the closeness repeat control (if necessary) by setting the same as well. There are no drawbacks in these approaches, except defining the integer $Q^{*}$ and tolerance $\delta$ is still an experience-based task. The integer $Q_{\max }$ is input externally by a decision-maker or an expert procedure organizer. Although, $Q_{\max }$ may be set sufficiently great when the procedure length is not limited.

The apparent merits of the developed algorithms are their simplicity and universality, ensuring algorithmic flexibility. The simple example with experts' estimations in the form of $2 \times 2$ matrices showed that these algorithms can be used in forming a matrix cluster. Therefore, they are going to form a criterion for a clusterization approach. Similarly to clusterization, the segment $\left[\lambda_{\min } ; \lambda_{\max }\right]$ is narrowed down to the segment $\left[\lambda_{\text {min }} ; \lambda_{\text {obs }}^{\langle 2\rangle}\right]$ or $\left[\lambda_{\text {min }} ; \lambda_{\text {obs }}^{\langle 3\rangle}\right]$ subject to the last obsolete value of $\lambda$, although a case $\lambda_{\mathrm{obs}}^{\langle i+1\rangle}=\lambda_{\mathrm{obs}}^{\langle i\rangle}$ occurs by inequality (4) after the inequalities (6) and (8). A parameter's confidence interval is thus determined. When narrowing, however, no reasons are available for moving the left end to the right. The exception for that comes if $\rho$ is closer and closer to $\lambda$ by keeping the inequality (5) true. By this type of expert estimation convergence, the segment $\left[\lambda_{\min } ; \lambda_{\max }\right]$ can be probably narrowed to

$$
\left[\frac{\lambda_{\text {min }}+\rho}{2} ; \lambda_{\text {obs }}^{\langle 2\rangle}\right]
$$

or similar. The said convergence of expert estimations does not have its definition now, but it is a matter of the impending continuation of research in adjusting the expert procedure parameters.

\section{CONClusion}

The adjustment removes the actual interval uncertainty by sliding down off the upper boundary and taking a portion of the expert estimations' average into the pivot point $\lambda$. The represented three algorithms can be applied in any study field involving expert estimations under unknown parameters. They are reversible, i. e., are easily inverted to reverse inequalities (1) and (6), implying sliding up off the boundary $\lambda_{\min }$. Hard adjusting is chosen for either less experienced procedures or a few $Q_{\max }$, and soft adjusting is chosen after good-enough expertise.

\section{REFERENCES}

[1] T. Frantti and M. Majanen, "An expert system for real-time traffic management in wireless local area networks," Expert Systems with Applicat., vol. 41, issue 10, pp. 4996-5008, Aug. 2014. https://doi.org/10.1016/i.eswa.2014.01.024

[2] J. Protasiewicz, W. Pedrycz, M. Kozłowski, S. Dadas, T. Stanisławek, A. Kopacz and M. Gałężewska, "A recommender system of reviewers and experts in reviewing problems," Knowledge-Based Systems, vol. 106, pp. 164-178, 2016. https://doi.org/10.1016/j.knosys.2016.05.041

[3] Y. Zhou, N. Fenton and M. Neil, "Bayesian network approach to multinomial parameter learning using data and expert judgments," Int. J. of Approximate Reasoning, vol. 55, issue 5, pp. 1252-1268, July 2014. https://doi.org/10.1016/j.ijar.2014.02.008

[4] G. Vessia, L. Pisano, C. Vennari, M. Rossi and M. Parise, "Mimic expert judgement through automated procedure for selecting rainfall events responsible for shallow landslide: A statistical approach to validation," Computers \& Geosciences, vol. 86, pp. 146-153, Jan. 2016. https://doi.org/10.1016/j.cageo.2015.10.015

[5] M. J. Kochenderfer, C. Amato, G. Chowdhary, J. P. How, H. J. Davison Reynolds, J. R. Thornton, P. A. Torres-Carrasquillo, N. K. Üre and J. Vian, Decision Making Under Uncertainty: Theory and Application. Cambridge, Massachusetts, London, England: The MIT Press, 2015.

[6] T. A. Saurin and S. S. Gonzalez, "Assessing the compatibility of the management of standardized procedures with the complexity of a sociotechnical system: Case study of a control room in an oil refinery," Applied Ergonom., vol. 44, issue 5, pp. 811-823, Sep. 2013. https://doi.org/10.1016/j.apergo.2013.02.003

[7] R. E. Walpole, R. H. Myers, S. L. Myers and K. Ye, Probability \& Statistics for Engineers \& Scientists, 9th ed. Boston, Massachusetts: Prentice Hall, 2012.

[8] Z. Xuan, H. Xia, and Y. Du, "Adjustment of knowledge-connection structure affects the performance of knowledge transfer," Expert Systems with Applicat., vol. 38, issue 12, pp. 14935-14944, Nov.-Dec. 2011. https://doi.org/10.1016/j.eswa.2011.05.054

[9] Y.-M. Wang, L.-H. Yang, Y.-G. Fu, L.-L. Chang and K.-S. Chin, "Dynamic rule adjustment approach for optimizing belief rule-base expert system," Knowledge-Based Systems, vol. 96, pp. 40-60, Mar. 2016. https://doi.org/10.1016/j.knosys.2016.01.003

[10] L. Barreira and C. Valls, "Robust nonuniform dichotomies and parameter dependence," J. of Math. Analysis and Applicat., vol. 373, issue 2, pp. 690-708, Jan. 2011. https://doi.org/10.1016/i.jmaa.2010.08.026

[11] D. Stoica, "Uniform exponential dichotomy of stochastic cocycles," Stochastic Processes and their Applicat., vol. 120, issue 10, pp. 1920-1928, Sep. 2010. https://doi.org/10.1016/i.spa.2010.05.016

[12] P. Ramazi, H. Hjalmarsson and J. Mårtensson, "Variance analysis of identified linear MISO models having spatially correlated inputs, with application to parallel Hammerstein models," Automatica, vol. 50, issue 6, 2014, pp. 1675-1683. https://doi.org/10.1016/j.automatica.2014.04.014

[13] O. J. Walch and M. C. Eisenberg, "Parameter identifiability and identifiable combinations in generalized Hodgkin-Huxley models," Neurocomputing, vol. 199, pp. 137-143, July 2016. https://doi.org/10.1016/j.neucom.2016.03.027

[14] A. Dembińska and N. Balakrishnan, "The asymptotic distribution of numbers of observations near order statistics," J. of Statistical Planning and Inference, vol. 138, issue 8, pp. 2552-2562, Aug. 2008. https://doi.org/10.1016/j.jspi.2007.10.023

[15] S. You, L. Hu, W. Mao, and X. Mao, "Robustly exponential stabilization of hybrid uncertain systems by feedback controls based on discrete-time observations," Statistics \& Probability Lett., vol. 102, pp. 8-16, July 2015. https://doi.org/10.1016/j.spl.2015.03.006

[16] Y. Song, X. Wang, L. Lei, and S. Yue, "Uncertainty measure for intervalvalued belief structures," Measurement, vol. 80, pp. 241-250, Feb. 2016. https://doi.org/10.1016/j.measurement.2015.11.032

[17] J. Wu, J. Gao, Z. Luo, and T. Brown, "Robust topology optimization for structures under interval uncertainty," Advances in Eng. Software, vol. 99, pp. 36-48, Sep. 2016. https://doi.org/10.1016/j.advengsoft.2016.05.002

[18] P. Révész, Z. W. Birnbaum, and E. Lukacs, The Laws of Large Numbers. New York, New York, London, England: Academic Press, 1968.

Vadim V. Romanuke was born in 1979. He received the degree of a Candidate of Technical Sciences and the degree of a Doctor of Technical Sciences in mathematical modeling and computational methods in 2006 and 2014, respectively. In 2016, he received the status of Full Professor.

V. V. Romanuke is a Professor at the Department of Applied Mathematics and Social Informatics of Khmelnitskiy National University. His research interests concern decision making, game theory, statistical approximation, and control engineering based on statistical correspondence.

Address: 11 Institutskaya Str., 29016, Khmelnitskiy, Ukraine.

E-mail: romanukevadimv@mail.ru 